\title{
Dermoscopy of halo nevus in own observation
}

\author{
Grażyna Kamińska-Winciorek ${ }^{1}$, Jan Szymszal ${ }^{2}$ \\ ${ }^{1}$ The Center for Diagnostics and Treatment of Skin Diseases, Katowice, Poland \\ Head of Center: Grażyna Kamińska-Winciorek MD, PhD \\ ${ }^{2}$ Department of Production Engineering, Silesian Technical University, Katowice, Poland \\ Head of Department: Teresa Lis Assoc. Prof, PhD
}

Postep Derm Alergol 2014; XXXI, 3: 152-158

DOI: 10.5114/pdia.2014.40963

\begin{abstract}
Introduction: Halo nevus $(\mathrm{HN})$ is a rare dermatologic entity characterized by a typical whitish rim encircling the existing melanocytic nevus resembling a halo. The clinical picture is suggesting its diagnosis, but so far only several dermoscopic descriptions of halo nevus have existed in the PubMed database.

Aim: To present the clinical and dermoscopic characteristics of halo nevus observed in dermoscopy.

Material and methods: Fifteen patients were diagnosed clinically and dermoscopically with halo nevus during planned routine dermoscopic examinations of all melanocytic lesions in 2007-2013. All digital images stored in the computer database were analyzed retrospectively according to the procedure described in the study. The clinical and dermoscopic parameters such as the dermoscopic pattern, color of nevus, special features and description of the surrounding halo were analyzed statistically.

Results: We analyzed 22 halo nevi (9 in females, 13 in males) in 15 patients ( 7 females, 8 males) diagnosed during the dermoscopic examination. The mean age of patients during dermoscopic examinations was 18.2 years. Mean patients' age at $\mathrm{HN}$ onset was 15.7 years. Halo nevi occurred the most often as a solitary lesion. The ratio of multiple halo nevi to solitary halo nevus was $5: 10$. Every third halo nevus was located on the posterior trunk upper. In $68.2 \%$ of HN cases, the surrounding rim (halo) was characterized by its homogenous, whitish color.

Conclusions: Dermoscopic patterns such as uniform globular and structureless constituted one-third each of them in all analyzed patterns.
\end{abstract}

Key words: dermoscopy, dermatoscopy, halo nevus, Sutton nevus.

\section{Introduction}

Halo nevus (HN) is a rare dermatologic entity characterized by a typical whitish rim encircling the existing melanocytic nevus resembling a halo [1]. In 1916, Sutton described halo nevus as a "leukoderma acquisitum centrifugum" [2]. Happle postulated that the term of Sutton nevus appears less appropriate than the alternative eponymic name as "Grünewald nevus" [2]. The clinical picture is suggesting its diagnosis, but so far only several dermoscopic descriptions of halo nevus have existed in the PubMed database [3-6]. Clinical and dermoscopic recognition of the whitish rim encircling the melanocytic lesion is a clue for differential diagnosis with melanoma presenting in some cases with regression.

A dermoscope is a stethoscope in the hand of the dermatologist and enables the doctor to best examine all skin lesions [7], especially to differentiate diagnostically difficult skin lesions [8, 9].

\section{Aim}

The aim of the study is to present the clinical and dermoscopic characteristics of halo nevus observed in dermoscopy in patients during a routine dermoscopic examination in the retrospective analysis of digital dermoscopic figures obtained.

\section{Material and methods}

Fifteen patients were diagnosed clinically and dermoscopically with halo nevus during the planned routine dermoscopic examination of all melanocytic lesions in the Private Dermatological Unit in 2007-2013. Halo nevus was defined as a melanocytic lesion with a surrounding whitish rim resembling a halo. Every halo nevus was checked with hand-held dermoscope Delta 20 Heine and then recorded as captured clinical and dermoscopic digital figures in FotoFinder Dermoscopie Dynamic Medicam

Address for correspondence: Grażyna Kamińska-Winciorek MD, PhD, The Center for Diagnostics and Treatment of Skin Diseases, 194/1 Armii Krajowej Av., 40-748 Katowice, Poland, phone/fax: +48 698626 208, e-mail: dermatolog.pl@gmail.com Received: 8.12.2013, accepted: 2.01.2014. 
500 and Medicam 800 HD (FotoFinder Systems GmbH). Captured images were analyzed retrospectively according to the procedure presented below.

All halo nevi were clinically and dermoscopically described taking into account their location and an overall dermoscopic pattern with a type of the surrounding halo.

Locations of halo nevus were divided according to Zalaudek [10] according to our own modification into: anterior trunk upper, middle and lower segments; posterior trunk upper, middle and lower segments; scalp; neck; nape; ear; face; upper limb: shoulder, antebrachial part; gluteal region; lower limb: thigh anterior and posterior, calf, tibial part; acral volar skin; anogenital region.

Dermoscopic images were analyzed according to the nomenclature proposed by other authors $[5,6,10]$ according to our own modification and halo nevi were dermoscopically subdivided into such subgroups as:

1. Uniform globular pattern or cobblestone,

2. Uniform reticular pattern,

3. Mixed pattern composed of central globules or mixed pattern composed of a structureless area surrounded by a network,

4. Mixed pattern composed of a central network or a structureless brown-gray area surrounded by a peripheral rim of small brown globules,

5. Starburst nevus,

6. Homogenous blue nevus,

7. Unspecific patterns: structureless non-pigmented or structureless brown and/or papillomatous structureless non-pigmented,

8. Blue granules and/or with scar-like areas,

9. Other networks,

10. Vascular (serpiginous, linear curved hair-pin, dotted vessels).

The color of the nevus was presented as: brown, light brown, white, pink, and black, gray, blue. Dermoscopic patterns of the halo of HN were divided into: whitish, bluish, brownish, café-au-lait, teleangiectotic and pinkish. Special features of HN include: a red halo occurring as "crown vessels", presence of an eczematous halo, a hemorrhage and central vessels as linear curved or serpiginous were taken into account.

The presence of a vitiligo and hypopigmented spots located according to HN location presented above was also examined.

\section{Statistical analysis}

The statistical assessment was performed using statistical software Statistica StatSoft version V 7.1 PL. In statistical analysis, the statistical level was $p<0.05$. In the statistical assessment, in the nominal scale the non-parametric chi-square test $\left(\chi^{2}\right)$ was used. To compare quantitative characteristics, parametric $t$ Student test or non-parametric $U$ Mann-Whitney rank sum test were adopted.

\section{Results}

Characteristics of the investigated patients' group

We analyzed 22 halo nevi (9 in females, 13 in males) in 15 patients ( 7 females, 8 males) diagnosed during the dermoscopic examination.

The mean age of patients during the dermoscopic examination was 18.2 years (median 14.9; SD 14.9 years), min. age was 8.7 and max. age -38.6 years. The patients' age at the HN onset was 15.7 years (median 13.1; SD 2.14 years); min. age of onset was 8.5, max. -39.1 years.

Table 1 shows general, clinical and dermoscopic characteristics of the investigated group of patients' with halo nevus. Patients consult a dermatologist because of the existing halo nevus 2 years after they had noticed first symptoms of the whitish rim. Table 2 presents the patients' age at HN onset in the history and patients' age of a first dermatological examination with a dermoscope in the basic diagnosis of HN.

\section{Location of halo nevus}

The most often location of analyzed $22 \mathrm{HN}$ were: posterior trunk upper - 7 lesions (31.8\%), posterior trunk middle -3 lesions (13.6\%) as well as anterior trunk upper and anterior trunk middle -3 lesions in each location (13.6\%). None of HN was located in anterior and posterior lower segments of the trunk. Halo nevi were found on the scalp in 2 patients only, all in men (9.1\%). Both in women and men, HN were located generally in the upper and middle segment of the anterior and posterior trunk (Table 3).

\section{Analysis of the dermoscopic pattern of halo nevus Overall general pattern of halo nevus}

The most common overall dermoscopic patterns (presented also in Table 1) were the uniform globular pattern (7/22; 31.8\%) and structureless pattern (7/22; 31.8\%). Blue granules and/or with scar-like areas with pattern coexisted in $3 / 22$ lesions (13.6\%) and only as a rest of network pattern in $3 / 22$ lesions (13.6\%). The cobblestone pattern concerned 2 lesions only (9.2\%). The most typical dermoscopic patterns of HN are presented in Figure 1.

\section{Halo nevus color}

In analyzed dermoscopic patterns of $22 \mathrm{HN}$ (Table 1) the most common color was a uniform color - mainly brown (6/22; 27.3\%), subsequently light-brown (4/22; 18.2\%) and gray $(3 / 22 ; 13.6 \%)$. Bicolor lesions occurred altogether in $40 \%$ of $\mathrm{HN}$, in 2 lesions in each color combination group: brown and light brown (2/22), brown and white (2/22), lightbrown and pinkish (2/22), light-brown and white (2/22).

\section{General pattern and halo nevus color}

The uniform globular pattern occurred generally in brown $(5 / 22 ; 22.7 \%)$, structureless in pinkish (2/22; $9.1 \%)$ and rest of network pattern in light brown (2/22; 9.1\%). 
Table 1. General, clinical and dermoscopic characteristics of the investigated group of patients' with halo nevus ( $F$ - female, $M$ - male)

\begin{tabular}{|c|c|}
\hline Number of patients & 15 (F: 7 (46.6\%), M: 8 (53.3\%)) \\
\hline Age of onset, mean, median, min., max. [years] & $15.7,13.1,8.5,39.1$ \\
\hline Age of inclusion, mean, median, min., max [years] & $18.2,14.9,8.7,38.6$ \\
\hline Fitzpatrick patients' type, $n(\%)$ & $1^{\text {st }}: 1(6.7 \%), 2^{\text {nd }}: 10(66.6 \%), 3^{\text {rd }}: 4(26.7 \%)$ \\
\hline Number of halo nevi & 22 (F: 9 (40.9\%), M: 13 (59.9\%)) \\
\hline Ratio of multiple halo nevi: solitary halo nevus & $5: 10$ \\
\hline Number of patients with multiple halo nevi & 5 with $12 / 22$ lesions \\
\hline Number of patients with solitary halo nevus & 10 with $10 / 22$ lesions \\
\hline \multicolumn{2}{|l|}{ Characteristics of the surrounding halo } \\
\hline Whitish & $15 / 22(68.2 \%)$ \\
\hline Whitish, telangiectatic & $4 / 15(18.2 \%)$ \\
\hline Pinkish, telangiectatic & $1 / 22(4.5 \%)$ \\
\hline Pinkish & $2 / 22(9.1 \%)$ \\
\hline \multicolumn{2}{|l|}{$\begin{array}{l}\text { Characteristics of halo nevus } \\
\text { Nevus color }\end{array}$} \\
\hline Brown & $6 / 22(27.3 \%)$ \\
\hline Light brown & $4 / 22(18.2 \%)$ \\
\hline Brown/light brown & $2 / 22(9.1 \%)$ \\
\hline Brown/white & $2 / 22(9.1 \%)$ \\
\hline Light brown/white & $1 / 22(4.5 \%)$ \\
\hline Light brown/pinkish & $2 / 22(9.1 \%)$ \\
\hline Pinkish & $2 / 22(9.1 \%)$ \\
\hline Gray & $3 / 22(13.6 \%)$ \\
\hline \multicolumn{2}{|l|}{ General pattern } \\
\hline Uniform globular & $7 / 22(31.8 \%)$ \\
\hline Cobblestone & $2 / 22(9.2 \%)$ \\
\hline Structureless non-pigmented or structureless brown & $7 / 22(31.8 \%)$ \\
\hline Rest of network & $3 / 22(13.6 \%)$ \\
\hline Rest of network with blue granules and/or with scar-like areas & $3 / 22(13.6 \%)$ \\
\hline \multicolumn{2}{|l|}{ Special features of halo nevus } \\
\hline None & $16 / 22(72.6 \%)$ \\
\hline Red halo "crown vessels" & $1 / 22(4.6 \%)$ \\
\hline Red halo "crown vessels" with central vessels & $2 / 22(9.1 \%)$ \\
\hline Hemorrhage with central vessels & $1 / 22(4.6 \%)$ \\
\hline Central vessels & $2 / 22(9.1 \%)$ \\
\hline
\end{tabular}

\section{General pattern and halo nevus location}

The uniform globular pattern was located mainly on the posterior trunk upper (3/22; $13.6 \%)$ and anterior trunk upper $(3 / 22 ; 13.6 \%)$. The structureless pattern occurred on the anterior trunk middle $(2 / 22 ; 9.1 \%)$ and posterior trunk middle (2/22; 9.1\%).

\section{General pattern and patient's age}

Three halo nevi with the rest of network pattern affected middle-aged patients (mean age 29 years; SD 12 years). Seven lesions with a structureless pattern occurred in patients with the mean age of 19.5 years (SD 7.2 years). Seven lesions with the uniform globular pat- 
Table 2. The patients' age at HN onset in the history and patients' age of the first dermatological examination with a dermoscope in basic diagnosis of $\mathrm{HN}$

\begin{tabular}{lcccccc}
\hline Patients' group $(n)$ & $\begin{array}{c}\text { Patients' age at HN onset } \\
\text { and age of basic HN } \\
\text { diagnosis }\end{array}$ & $\begin{array}{c}\text { Mean age } \\
\text { [years] }\end{array}$ & $\begin{array}{c}\text { Median } \\
\text { [years] }\end{array}$ & $\begin{array}{c}\text { Min. } \\
\text { [years] }\end{array}$ & Max. [years] & SD \\
\hline Female (7) & Age of onset & 15.7 & 12.6 & 8.5 & 39.1 & 10.5 \\
\cline { 2 - 7 } & Age of diagnosis & 18.5 & 14.3 & 8.7 & 38.5 & 11.5 \\
\hline Male (8) & Age of onset & 15.7 & 14.7 & 8.9 & 26.0 & 6.5 \\
\cline { 2 - 7 } & Age of diagnosis & 17.9 & 19.1 & 9.2 & 26.1 & 6.2 \\
\hline
\end{tabular}

Table 3. Location of HN depending on gender

\begin{tabular}{|c|c|c|c|c|c|c|c|c|c|}
\hline & $\begin{array}{c}\text { Anterior } \\
\text { trunk upper }\end{array}$ & $\begin{array}{c}\text { Anterior } \\
\text { trunk middle }\end{array}$ & $\begin{array}{c}\text { Posterior } \\
\text { trunk upper }\end{array}$ & $\begin{array}{c}\text { Posterior } \\
\text { trunk middle }\end{array}$ & Scalp & Neck & Nape & Face & In all \\
\hline Female & 2 & 2 & 2 & 1 & 0 & 0 & 1 & 1 & 9 \\
\hline$\%$ column & 66.67 & 50.00 & 28.57 & 33.33 & 0.00 & 0.00 & 100.00 & 100.00 & \\
\hline$\%$ line & 22.22 & 22.22 & 22.22 & 11.11 & 0.00 & 0.00 & 11.11 & 11.11 & \\
\hline Male & 1 & 2 & 5 & 2 & 2 & 1 & 0 & 0 & 13 \\
\hline$\%$ column & 33.33 & 50.00 & 71.43 & 66.67 & 100.00 & 100.00 & 0.00 & 0.00 & \\
\hline$\%$ line & 7.69 & 15.38 & 38.46 & 15.38 & 15.38 & 7.69 & 0.00 & 0.00 & \\
\hline \multirow[t]{2}{*}{ In all } & 3 & 4 & 7 & 3 & 2 & 1 & 1 & 1 & 22 \\
\hline & $13.64 \%$ & $18.18 \%$ & $31.82 \%$ & $13.64 \%$ & $9.09 \%$ & $4.55 \%$ & $4.55 \%$ & $4.55 \%$ & \\
\hline
\end{tabular}

$\chi^{2}=8.208, p=0.3146$

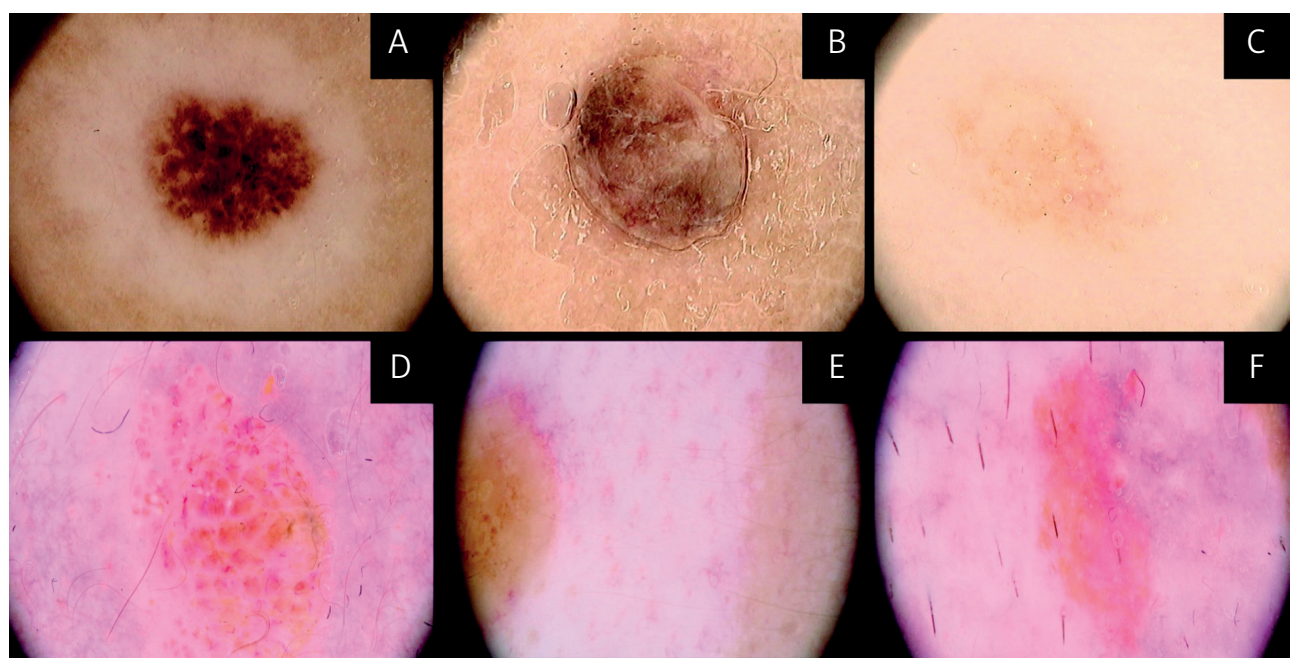

Figure 1. Dermoscopic pictures of halo nevus in our own study. Figure presents the most common dermoscopic patterns with selected dermoscopic features of halo nevus in investigated patients' group. A - Uniform globular pattern in brown with a typical surrounding depigmented whitish halo. B - Structureless pattern in brown-white. C - Blue granules and a rest of network pattern of clinically disappearing halo nevus. D - Cobblestone pattern with several central serpiginous vessels. E - Halo nevus with circumflexing linear vessels like a "vascular crown". There is seen a very sharply demarcated rim in slight pinkish in contrast with normal skin in light-brown. F - Halo nevus with circumflexing linear vessels like a "vascular crown" and several serpiginous vessels inside the nevus. The surrounding halo is telangiectatic and pinkish 


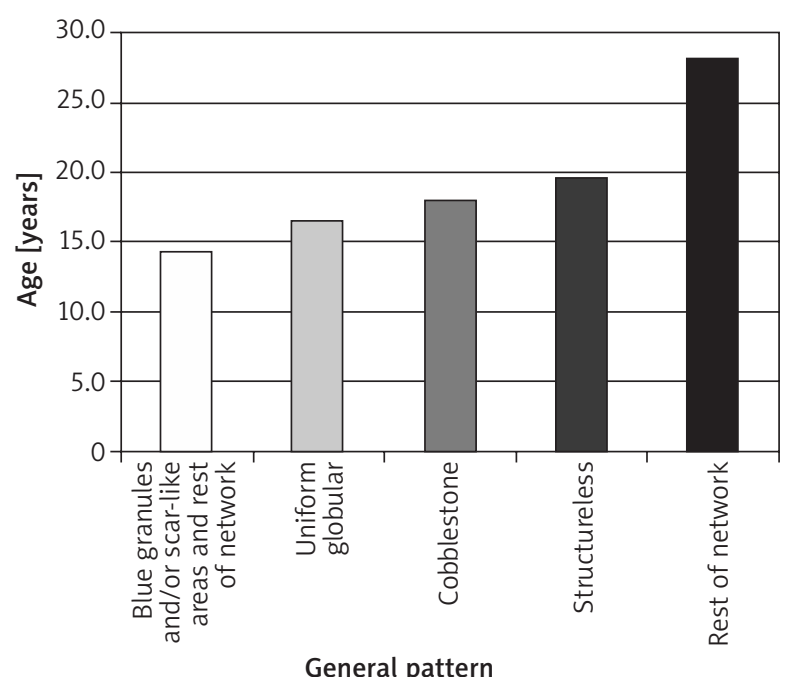

Figure 2. Dermoscopic patterns of HN depending on the patients' age

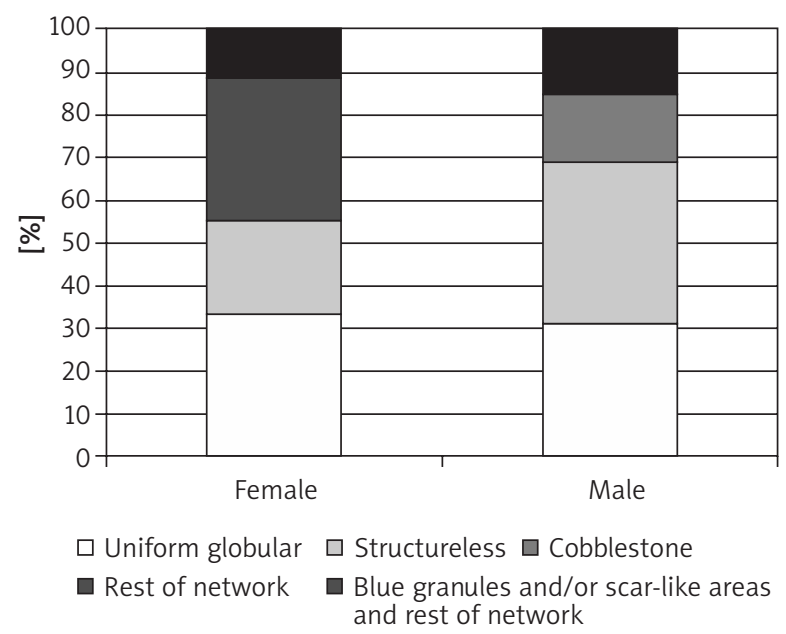

Figure 3. Dermoscopic patterns of $\mathrm{HN}$ depending on the patients' gender

tern concerned adolescents with the mean age of 16.6 years (SD 7.17 years). Occurrence of selected dermoscopic patterns depending on the patients' age is shown in Figure 2.

\section{General pattern of halo nevus and gender}

The dermoscopic pattern described as rest of network affected females only (3) whereas the cobblestone pattern entirely males. Dermoscopic patterns such as the uniform globular pattern (female $(F)-3$, male $(M)-4$ ) and blue granules and/or with scar-like areas and rest of network ( $F-1, M-2)$ occurred in both females and males. Occurrence of dermoscopic patterns depending on gender is presented in Figure 3.

\section{General pattern and special dermoscopic features of halo nevus}

In the analysis of special features concerning dermoscopic patterns of HN, more than half of halo nevi $(16 / 22)$ showed no special features ( $72.6 \%$ all of HN). The red halo described as "crown vessels" concerned 3 lesions only, all in the structureless dermoscopic pattern.

\section{Surrounding rim (halo) of halo nevus}

In the majority of cases (68.2\%), halo nevi were characterized by occurrence of a whitish, homogenous halo (15/22 lesions) (Table 1, Figure $1 \mathrm{~A})$. Overall in 4 lesions in their whitish halo the telangiectasias were also visible (18.2\%) (Figure $1 \mathrm{~F})$, which in 1 nevus coexisted with a pinkish halo (1/11; $4.5 \%)$. In $2 \mathrm{HN}$ only, a pinkish surrounding halo was visible (9.1\%) (Figure $1 \mathrm{E})$.

\section{Surrounding rim of halo nevus and special dermoscopic features of halo nevus}

Twelve of 22 halo nevi (54.5\%) revealed no special features present in the case of a whitish halo occurrence $\left(\chi^{2}\right.$ test $\left.p=0.033\right)$. Four of 22 lesions did not have any special features in the case of a whitish and telangiectatic halo. In all cases of the pinkish, and telangiectatic and pinkish halo, special features covering a red halo ("crown vessels") (Figure 1 E) with central (serpiginous) vessels (Figure $1 \mathrm{~F}$ ) accompanied this surrounding rim.

\section{Halo nevus and white hypopigmented spots/vitiligo}

No halo nevus located in the anterior trunk upper appeared with a spot and vitiligo. Halo nevi located in the anterior trunk middle occurred with hypopigmented spots.

Hypopigmented spots located in the posterior trunk middle occurred with halo nevi located in the anterior trunk upper (3). Hypopigmented spots occurring in the posterior trunk middle also coexisted with halo nevus situated in the same location - the posterior trunk middle (2) and in two nevi located in the posterior trunk upper.

\section{Discussion}

Regarding the current dermoscopic proposal of nevus classification [6], halo nevus is included into groups of nevi with special features, among combined nevi, irritated nevi, nevi with an eczematous halo, recurrent nevi and unclassifiable nevi [6]. Halo nevus is a congenital or acquired nevus, developing a rim of depigmentation surrounding the melanocytic central part (monomorphous brown to gray) [6]. In dermoscopy it is characterized by occurrence of the globular pattern with blue pepper-like granules and/or white-scar areas [5, 6]. In our study we analyzed $22 \mathrm{HN}$ in 15 patients. Previously published original data included 9 patients with $9 \mathrm{HN}$ in Schwartz et al. study [3] and $138 \mathrm{HN}$ in 87 patients in Kolm et al. observation [4]. The patients' phototype according to 
Fitzpatrick in our study was in favor of the $2^{\text {nd }}(66.6 \%)$ vs. the $3^{\text {rd }}(33.3 \%)$ in Schwartz' observation [3]. Uniform globular and structureless brown patterns were the most often in our observation. A previous study on the dermoscopic picture of $\mathrm{HN}$ also found the homogenous-globular pattern as the most frequent followed in frequency by the homogenous and globular patterns [4]. Homogenous and globular patterns were the most common with a dominant reticular pattern in a previous dermoscopic study [2]. Blue granules and/or with scar-like areas and rest of network patterns in our own observation constituted the third most often dermoscopic pattern of $\mathrm{HN}$, especially found in patients with the mean age of 29 years.

The mean age of HN onset in our study was 15.7 years, but in Patrizi et al. study [11] the mean age of onset was 8 years. The mean age of patients with $\mathrm{HN}$ analyzed by dermoscopy was 18 years in our study, in whereas in Schwartz's et al. [3] and Kolm's et al. [4] study the mean age was respectively 19 an 22 years) [3, 4]. The most typical location of HN was the posterior trunk [3], and generally the back [4] similar to our observation. Halo nevus occurred mainly as a solitary lesion [11], what was also shown in our conducted study. Multiple clinical presentation of $\mathrm{HN}$ is rather rare, and such manifestation suggests a higher risk of vitiligo and autoimmune disease development comparing to the solitary lesion [6]. The highest number of halo nevi in one patient including 28 lesions was described by Ohtsuka in 2009 [12].

The most common dermoscopic patterns such as globular and structureless ones can reflect the age of the patients with $\mathrm{HN}$ and they belong to the most common pattern of melanocytic nevi. According to Zalaudek et al. [13], globular or structureless patterns are the most typical for children [13]. These patterns also prevail on the upper trunk and on the head/neck location [13]. Color of the nevus depends on Fitzpatrick phototype and in fair skin the most popular color is light brown [14].

In our study, $68.2 \%$ of cases of HN surrounded by a depigmented rim (halo) was characterized by its whitish color. In these cases of HN, the depigmented white rim is called regression-like depigmentation [15]. The red halo as crown vessels with central vessels (serpiginous) was accompanied always by a pinkish and telangiectatic-pinkish halo. In Kolm et al. [4] study, only in several $\mathrm{HN}$ cases, a light brown to pink central area with dotted vessels was seen [4]. Sometimes the central component of the nevus may disappear leaving reddish, central pigmentation with occurrence of vessels derived from the dermal venous plexus [4, 15].

None of the presented HN cases in our own study was associated with vitiligo. Patrizi et al. [11] suggested two different types of halo nevus in children: transient benign phenomena without any correlation with autoimmune disease, which is rather single and spontaneously regressive, and the second one combined with multiple halo nevi, which indicated an association with vitiligo, autoimmune disease and positive family history [11].

Van Geel et al. [16] identified a subset of halo nevi with additional subtle depigmentations distant to HN and called "halo nevi associated-leukoderma" [16]. In our own study, several depigmented spots were found, especially in the posterior trunk middle related to halo nevi located in the anterior trunk upper (3/22), but the biopsy from spots has been not taken.

We should be aware that in patients with halo nevus and/or vitiligo and/or leukoderma, the entire dermoscopy examination should be performed to exclude the existing melanoma [17]. An immune response to melanocyte differentiation antigen to transformed and normal melanocytes manifests clinically at distant sites as melanoma-associated vitiligo or leukoderma and halo nevi [14].

A limitation of the study is the low number of patients involved in observations, which is a result of rare occurrence of this dermatological entity.

\section{Conclusions}

Halo nevi occurred most often as a solitary lesion. One third of HNs were located on the posterior trunk upper. Halo nevi never occurred at the anterior and posterior lower segments of the trunk. In $68.2 \%$ of HN, the surrounding rim (halo) was characterized by its homogenous, whitish color. Dermoscopic patterns such as uniform globular and structureless constituted one-third each of them in all analyzed patterns. Blue granules and/or with scar-like areas and rest of network patterns were the third most common dermoscopic pattern of HN.

\section{Conflict of interest}

Authors declare no potential conflict of interest. Funding sources: none.

\section{References}

1. Pustisek N, Sikanić-Dugić N, Hirsl-Hećej V, et al. "Halo nevi" and UV radiation. Coll Antropol 2010; 34 Suppl. 2: 295-7.

2. Happle R. Grunewald nevus. Hautarzt 1994; 45: 882-3.

3. Schwartz RJ, Vera K, Navarrete N, Lobos P. In vivo reflectance confocal microscopy of halo nevus. J Cutan Med Surg 2013; 17: 33-8.

4. Kolm I, Di Stefani A, Hofmann-Wellenhof R, et al. Dermoscopy patterns of halo nevi. Arch Dermatol 2006; 142: 1627-32.

5. Gulia A, Massone C. Advances in dermoscopy for detecting melanocytic lesions. F1000 Med Rep 2012; 4: 11.

6. Argenziano G, Zalaudek I, Ferrara G, et al. Proposal a new classification system for melanocytic nevi. BJD 2007; 157: 217-27.

7. Zalaudek I, Lallas A, Moscarella E, et al. The dermatologist's stethoscope-traditional and new applications of dermoscopy. Dermatol Pract Concept 2013; 3: 67-71.

8. Kamińska-Winciorek, Śpiewak R. Dermoscopy on nevus comedonicus; a case report and review of the literature. Postep Derm Alergol 2013; 30: 252-4. 
9. Kamińska-Winciorek, Właszczuk P, Wydmański JD. "Mistletoe sign" probably a new dermoscopic descriptor for melanoma in situ and melanocytic junctional nevus in the inflammatory stage. Postep Derm Alergol 2013; 30: 316-9.

10. Zalaudek I, Schmid K, Marghoob AA, et al. Frequency of dermoscopic nevus subtypes by age and body site: a cross-sectional study. Arch Dermatol 2011; 147: 663-70.

11. Patrizi A, Bentivogli M, Raone B, et al. Association of halo nevus/i and vitiligo in childhood: a retrospective observational study. JEADV 2013; 27: e148-52.

12. Ohtsuka T. Multiple sutton nevi: hypomelanocytic halo development around 28 melanocytic nevi. J Dermatol 2009; 36: 355-7.

13. Zalaudek I, Cacitrala C, Moscarella E, et al. What dermoscopy tells about nevogenesis? J Dermatol 2011; 38: 16-24.

14. Zalaudek I, Argenziano G, Mordente I, et al. Nevus type in dermoscopy is related to skin type with white persons. Arch Dermatol 2007; 143: 351-6.

15. Larre-Borges A, Zalaudek I, Longo C, et al. Melanocytic nevi with special features: clinical-dermoscopic and reflectance confocal microscopic - findings. JEADV 2013; DOI: 10.1111/ jdv.12291.

16. Van Geel N, Speeckeart R, Lambert J, et al. Halo nevi with associated vitiligo-like depigmentations: pathogetic hypothesis. JEADV 2012; 26: 755-61.

17. Naveh HP, Rao UN, Butterfield LH, et al. Melanoma-associated leukoderma - immunology in black and white? Pigment Cell Melanoma Res 2013; 26: 796-804. 